\title{
Characterization and Evaluation of Silk Protein Hydrogels for Drug Delivery
}

\author{
Jia-You FAnG, ${ }^{*}, a$ Jyh-Ping Chen, ${ }^{b}$ Yann-Lii Leu, ${ }^{c}$ and Hsin-Yuan $\mathrm{WANG}^{a}$ \\ ${ }^{a}$ Pharmaceutics Laboratory, Graduate Institute of Natural Products, Chang Gung University; ${ }^{b}$ Graduate Institute of \\ Biochemical and Biomedical Engineering, Chang Gung University; and ${ }^{c}$ Natural Products Laboratory, Graduate Institute \\ of Natural Products, Chang Gung University; Kweishan, Taoyuan, Taiwan. \\ Received July 27, 2005; accepted October 31, 2005
}

The objective of this study was to characterize and evaluate the physicochemical properties and drug release profiles of hydrogels composed of silk protein (SP) polymers. SPs with a low MW (SPL, ca. $18 \mathrm{kDa}$ ) and a high MW (SPH, ca. $76 \mathrm{kDa}$ ) were used for preparing hydrogels. Both the random coil form and $\beta$-sheet conformation simultaneously existed in the hydrogels according to Fourier-transformed IR determination. Morphologically, the hydrogels showed a sponge-like cross-linked structure produced by physical entanglement as well as chemical hydrogen and covalent bindings. The in vitro buprenorphine delivery from SPH hydrogels showed a slow-release effect, and a zero-order rate was obtained for all preparations. Drug release could be controlled by varying the SPH concentrations or incorporation of SPL into the systems. SP hydrogels showed a stronger barrier property for hydrophilic solutes than for hydrophobic solutes. The incorporation of SPH into Pluronic F-127 (PF-127) hydrogels changed the gel structure from amorphous micelles to a regularly interconnected texture with pores. Furthermore, SPH as an adjuvant polymer in PF-127 and chitosan hydrogels lowered and controlled the amount of drug released from those systems.

Key words silk protein polymer; controlled release; hydrogel; buprenorphine; drug delivery

Silk is generally defined as protein polymers that are spun into fibers by some lepidopteran larvae. The most extensively characterized silks are from the domesticated silkworm (Bombyx mori). ${ }^{1)}$ Because of their impressive mechanical properties, biocompatibility, and biodegradable features, silk proteins (SPs) provide an important set of material options in the fields of controlled release, biomaterials, and scaffolds for tissue engineering. ${ }^{2)}$ One important material option for biomaterials such as SPs is the formation of hydrogels. The design and development of injectable drug delivery systems capable of forming hydrogel implant matrices has been the focus of much research. ${ }^{3-5}$ )

Knowledge of solute transport mechanisms in hydrogels is crucial for many biomedical applications including tissue engineering, cell delivery, and drug delivery systems. Buprenorphine is a morphine-like drug with partial agonist activity at the $\mu$-opiate receptor and antagonist activity at the $\kappa$-opiate receptor. It is approximately 100 times more potent than morphine and has been widely used in the treatment of acute and chronic pain. ${ }^{6,7)}$ Its half-life after parenteral administration is estimated to be $3-5 \mathrm{~h}$, and the recommended dosing frequency is 3 or 4 times daily. ${ }^{8}$ ) To circumvent these unfavorable biopharmaceutical characteristics and to improve the therapeutic effectiveness in pain management, it is desirable to develop prolonged-release delivery systems for buprenorphine. The aim of this study was to assess the feasibility of SP hydrogels as a controlled-release vehicle for buprenorphine. The physicochemical properties of the hydrogels were characterized by Fourier-transformed IR (FTIR), scanning electron microscopy (SEM), and viscosity. In addition to buprenorphine, the diffusivities from SP hydrogels of other solutes, such as nalbuphine, its prodrug, fluorescein isothiocyanate (FITC), and FITC-labeled dextran (FD), were also examined to explore the release mechanisms. SPs were also used as an adjuvant polymer for Pluronic F-127 (PF-127) and chitosan hydrogels in this present study.

\section{Experimental}

Materials Buprenorphine hydrochloride was purchased from Macfarlan Smith (U.K.). FITC and FD with average MWs of $4.4 \mathrm{kDa}$, urea, and Pluronic F-127 (PF-127) were obtained from Sigma Chemical (St. Louis, MO, U.S.A.). Chitosan (Chitosan $100^{\circledR}$ ) was purchased from Wako Chemical (Osaka, Japan). Nalbuphine and nalbuphine propionate were synthesized and supplied by the School of Pharmacy, National Defense Medical Center (Taipei, Taiwan). Protein polymers from silk with a high MW (SPH, average MW of $76 \mathrm{kDa}$ ) and a low MW (SPL, average MW of $18 \mathrm{kDa}$ ) were kindly provided by Aotesi Biochemical Co. (Shanghai, China).

Preparation of Hydrogels For preparing the SP hydrogels, the SP was added into $\mathrm{pH} 4$ citrate-phosphate buffer, and the mixture was continuously stirred on a hot plate set to $60^{\circ} \mathrm{C}$ for $4 \mathrm{~h}$. Aqueous buffer at $\mathrm{pH} 4$ was chosen because the decrease in $\mathrm{pH}$ might decrease the repulsion among SP molecules and promote the desorption of water from the proteins, resulting in shorter gelation times. ${ }^{1)}$ The mixture was then stirred at room temperature for $30 \mathrm{~min}$, while the model drug was incorporated into the mixture (10.7 mM buprenorphine, $1.4 \mathrm{~mm}$ nalbuphine and its prodrug, or $250 \mu \mathrm{M}$ for FITC and FD). All the chemical structures of model drugs used in this study are demonstrated in Fig. 1. This mixture was refrigerated at $4{ }^{\circ} \mathrm{C}$ for $20 \mathrm{~h}$ to form the rigid hydrogels.

To prepare the PF-127 hydrogels, the required amounts of polymer and drug were dispersed in $\mathrm{pH} 4$ buffer. The partially dissolved PF-127 solution was stored in a refrigerator $\left(4^{\circ} \mathrm{C}\right)$ until all of the polymer had completely dissolved (approximately $24 \mathrm{~h}$ ). To prepare the chitosan hydrogels, half of the calculated amount of polymer was added to an aqueous solution with $3 \%$ $(\mathrm{w} / \mathrm{v})$ lactic acid, after which the mixture was stored at $4{ }^{\circ} \mathrm{C}$ for $24 \mathrm{~h}$. The residual lactic acid-containing solution and drug were added to the mixture with continuous stirring for $1 \mathrm{~h}$.

The procedures for manufacturing the blended PF-127/SP and chitosan/SP hydrogels were similar to the preparation of hydrogels with SP alone. PF-127 or chitosan was added before storage in the refrigerator at $4^{\circ} \mathrm{C}$.

FTIR Absorption Spectroscopy These measurements were carried out on a Jasco FT/IR-410 FTIR spectrometer (Tokyo, Japan) using the attenuated total reflection (ATR) method. Germanium crystals were used as an ATR crystal. The hydrogels were inserted and stuck to the ATR crystal plates.

Scanning Electron Microscopic (SEM) Examination Hydrogels were frozen at $-80{ }^{\circ} \mathrm{C}$ and then lyophilized by a freeze-drying method. Samples were fractured in liquid nitrogen and examined using a Hitachi S-2400 SEM (Tokyo, Japan). All analyses were performed in a blinded fashion.

Viscosity Measurements The viscosity of the hydrogels was deter- 
mined using programmable viscometer (Model DV-II +, Brookfield, Middleboro, MA, U.S.A.). The T-bar spindle (code number: T-D) was used for the determination. Readings were recorded $30 \mathrm{~s}$ after the measurement was made, when the level had stabilized.

In Vitro Drug Release The in vitro release studies were performed using Franz diffusion cells. A cellulose membrane (CelluSep ${ }^{\circledR}$, Membrane Filtration Products, Seguin, TX, U.S.A.) with a MW cutoff of $6000-8000$ was mounted between the donor and receptor compartments. The donor phase consisted of $1 \mathrm{~g}$ of hydrogel with various drugs. The top of the donor compartment was covered with parafilm. The receptor medium consisted of $10 \mathrm{ml}$ of $\mathrm{pH} 6$ citrate-phosphate buffer for buprenorphine and $\mathrm{pH} 7.4$ buffer for the other solutes. The available diffusion area was $1.54 \mathrm{~cm}^{2}$. The stirring rate of the receptor was $600 \mathrm{rpm}$, and the temperature was maintained at $37^{\circ} \mathrm{C}$. At appropriate intervals, $300-\mu 1$ aliquots of the receptor medium were withdrawn and replaced with fresh buffer. Samples of buprenorphine, nalbuphine, and nalbuphine propionate were analyzed by HPLC as described earlier. $^{7,9)}$ Samples of FITC and FD were analyzed using a Hitachi F-2500 fluorescence spectrometer at an excitation wavelength of $494 \mathrm{~nm}$ and emission wavelength of $520 \mathrm{~nm}$.

Statistical Analysis The statistical analysis of differences between different formulations was performed using the unpaired $t$-test. A 0.05 level of probability $(p<0.05)$ was taken as the level of significance. An analysis of variance (ANOVA) test was also used.

\section{Results and Discussion}

Physicochemical Properties of SP Hydrogels The SPs used in this present study were polymers whose compositions are predominantly amino acids. The SPH showed high contents of serine $(28.91 \%)$, aspartic acid (19.96\%), and glutamine $(13.68 \%)$ as analyzed on a Hitachi $835-50$ amino acid analyzer (reports from Zhejiang Silk Science Research Institute, Zhejiang, China, Oct. 1, 2002). The purity of the proteins in this product was $>90 \%$. The SPL of a lower MW was hydrolyzed from the SPH. The SPL showed similar amino acid contents with the SPH $(27.50 \%$ serine, $17.84 \%$ aspartic acid, $11.86 \%$ glutamine, etc.). The SPH at $10 \%$ in $\mathrm{pH} 4$ buffer formed a semisolid hydrogel. The 3-dimensional structure of the hydrogels is often described as a mesh, with the spaces between the polymer chains filled with water. ${ }^{10)}$ The hydrogel is defined as the polymers which have the ability to swell in water or aqueous solvent systems. The polymer structure is able to retain the solvents forming a swollen gel phase and in cross-linked systems. ${ }^{11)}$ As water penetrates the polymer, swelling occurs and the gel of polymer in the rubbery state is formed. ${ }^{12)}$ On the other hand, a $10 \%$ SPL does not produce the semisolid form because of its low MW.

The SPH hydrogels were examined using FTIR to determine the conformational changes in the SPs. As shown in Fig. 1A, the SPH exhibited absorption bands at 1648 (amide I), 1557 (amide II), and $1241 \mathrm{~cm}^{-1}$ (amide III) which are attributed to the random coil conformation, while the absorption bands at 1638 (amide I), 1543 (amide II), and $1262 \mathrm{~cm}^{-1}$ (amide III) can be attributed to the $\beta$-sheet structure, indicating a mixture of random coil and $\beta$-sheet structures. ${ }^{13,14)}$ The SPL showed a similar IR absorption pattern with the SPH, except for the minor absorption at the amide II peaks (Fig. $2 \mathrm{~A}$ ). The mixture of the random coil and $\beta$-sheet conformations was maintained after blending the SPH and SPL. The presence of the $\beta$-sheet structure promotes the stability of silk hydrogels and forms the rigid structure in water. ${ }^{1)}$ However, the crystalline characteristics of the $\beta$-sheet may slow the degradation in the body. ${ }^{2)}$ Its coexistence with the random coil form may partially resolve this problem.

The freeze-dried forms of the hydrogels were prepared to examine the SEM appearance. The SEM results showed that
(A)

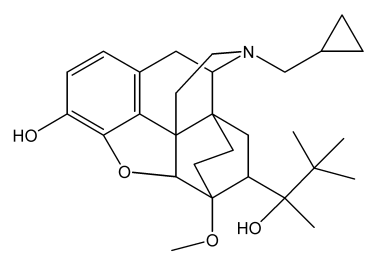

(C)

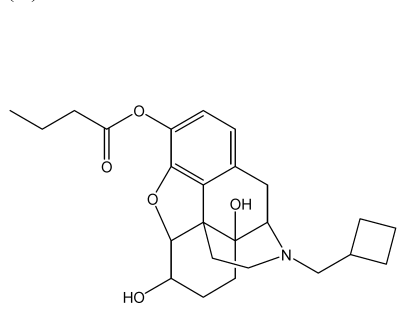

(B)

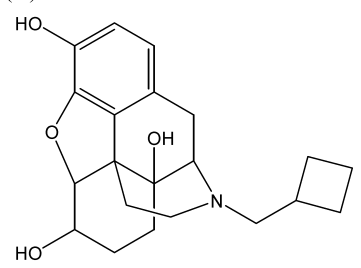

(D)
(E)

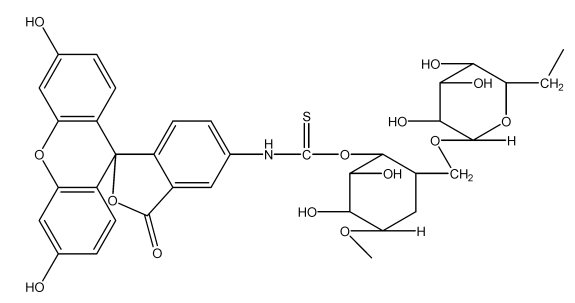

Fig. 1. The Chemical Structures of Buprenorphine (A), Nalbuphine (B), Nalbuphine Propionate (C), Fluorescein Isothiocyanate (FITC) (D), and Dextran (Which Is Conjugated with FITC at a MW of $4.4 \mathrm{kDa}$ Used in This Study) (E)

the hydrogel composed of the SPH had a network and sponge-like structures (Fig. 3A). The micrograph shows that the spherical pores were well interconnected throughout the scaffold matrix. The white portion is SP polymers and the black portion is the space occupied by the water molecules if the polymer had been hydrolyzed with water (before freezedrying). Hydrogels containing both the SPH and SPL exhibited porous structures composed of sheets of polymers with interconnecting channels on the basis of the SEM analysis (Fig. 3B). The average diameter of the pores seemed to decrease, and pore density increased with the addition of SPL in the hydrogel composed of SPH.

Gel formation was further traced by the viscosity of the formulations. Gelation occurs because of the formation of inter- and intramolecular interactions among the protein chains, including hydrophobic interactions and hydrogen bonds. With an increase in the SPH content of from $8 \%$ to $12 \%$, interactions among the SP chains increased, resulting in an increase in the viscosity (Table 1). SP molecules are thereby able to interact more readily, leading to physical cross-links. The incorporation of SPL from $2 \%$ to $6 \%$ did not influence the viscosity of the hydrogels composed of SPH $(p>0.05)$. This may indicate that the increased cross-linkages shown in the SEM of SPH hydrogels blended with the SPL did not further increase the gelation degree. Cross-links can be formed by covalent and ionic bonds, as well as by van der Waals forces, physical entanglement, and hydrogen bonds. ${ }^{10)}$ Urea is known to denature proteins by disrupting and pre- 
(A)

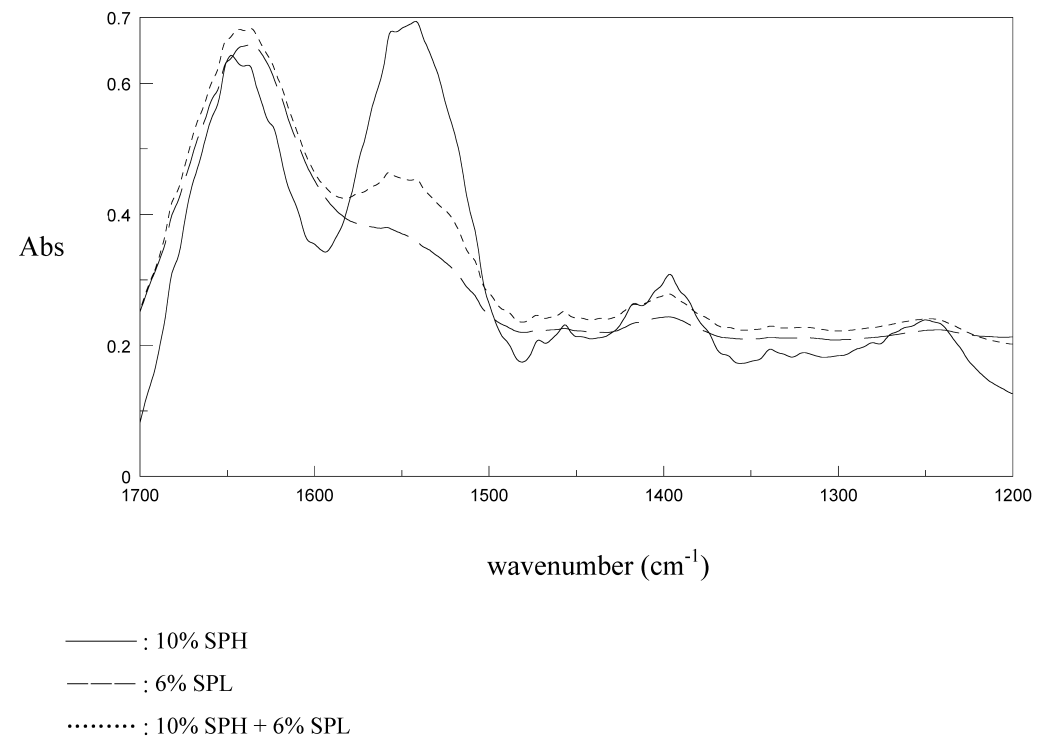

(B)

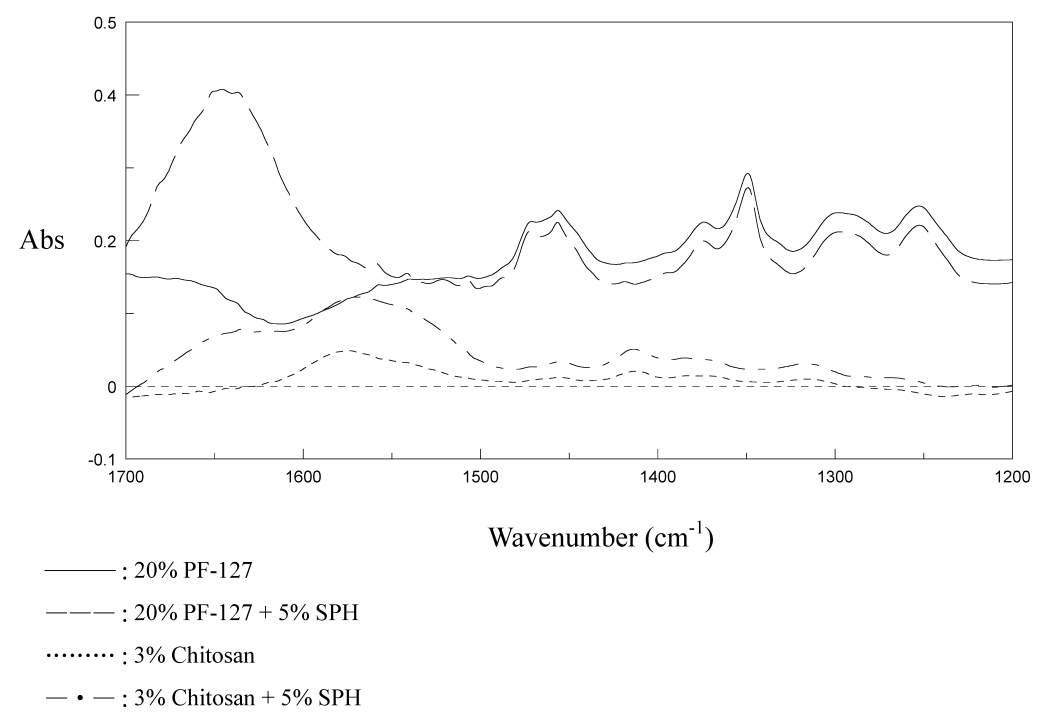

Fig. 2. FTIR Spectra of SP Hydrogels (A) and PF-127/Chitosan Hydrogels with or without SPH (B)

venting the formation of hydrogen bonds. If hydrogen bond generation or crystallization is the mechanism by which SP gels form, urea should retard or inhibit gelation. ${ }^{15)}$ As shown in Fig. 4A, the addition of urea gradually decreased the viscosity of the hydrogels composed of the SPH. Random coils of the protein polymers in the aqueous solution are changed into a $\beta$-sheet structure due to the intermolecular hydrogen binding. ${ }^{16)}$ SPH hydrogels retained their high viscosity when urea was added at lower concentrations, suggesting that other forces other than hydrogen bonds are responsible for the cross-linkages. Covalent bonds and physical entanglement are other possible interactions.

The addition of $1 \mathrm{M}$ urea produced a steep reduction in the viscosity of blended SPH and SPL hydrogels (Fig. 4A). Further increments of urea in gels only slightly further decreased the viscosity. The viscosity data of the hydrogels with SPH as well as with combined SPH and SPL were almost the same. This possibly indicates the importance of hydrogen bindings in the interactions between the SPH and SPL. This may be the reason why the addition of the SPL did not further increase the viscosity of SPH hydrogels since the hydrogen bonding is a weak force for cross-linking.

Drug Release from SP Hydrogels Figure 5A shows the in vitro release profile of buprenorphine from a $\mathrm{pH} 4$ aqueous solution. An initial rapid release of buprenorphine in the first $3 \mathrm{~h}$ was followed by retarded release until the end of the experiment. After calculation, there was only $c a$. $4 \%$ drug dose released from aqueous solution to the receptor phase. That is, most of the buprenorphine molecules remained in the donor after a 4-h administration. The limited release amount may be due to the use of in vitro Franz cell. Since drugs may release to a definitive space of receptor $(10 \mathrm{ml}$ in this study), the drug loading in the receptor medium is limited. SPH hydrogels exhibited slower release of buprenorphine compared to the aqueous solution $(p<0.05)$. The release kinetics were most adequately described as being zero order $(r>0.99)$ with respect to the molecules released. A constant rate of buprenorphine release was also observed for the hydrogels. 
This result possibly indicates that the drug released from SPH gels was diffusion-controlled through the matrix. Hydrogels containing $12 \% \mathrm{SPH}$ released buprenorphine more slowly than did hydrogels containing $8 \%$ and $10 \%$ polymer $(p<0.05)$. An increase in the polymer concentration resulted in an increase in polymer-polymer interactions and cross-
(A)

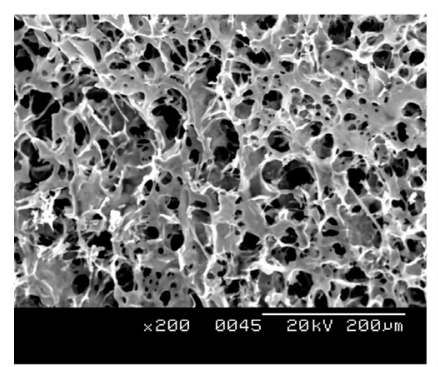

(C)

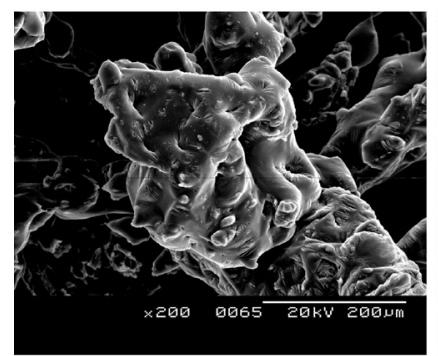

(E)

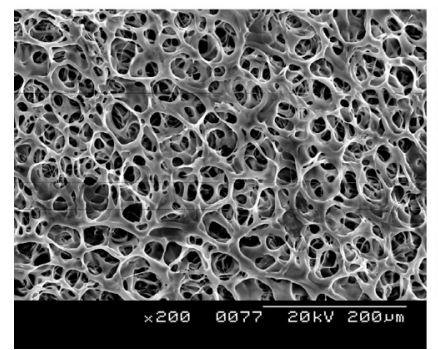

(B)

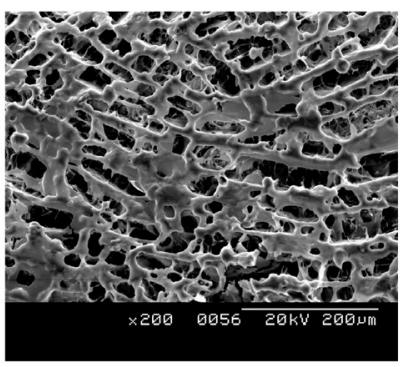

(D)

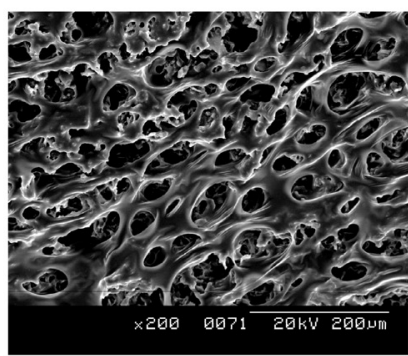

(F)

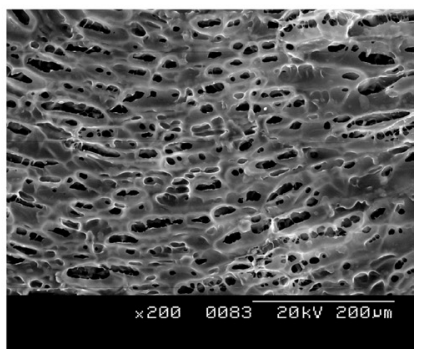

Fig. 3. SEM Images (Magnification, $\times 200)$ of Hydrogels after FreezeDrying Process Composed of $10 \%$ SPH (A), $10 \%$ SPH and $6 \%$ SPL (B), $20 \%$ PF-127 (C), 20\% PF-127 and 5\% SPH (D), 3\% Chitosan (E), and 3\% Chitosan and $10 \% \mathrm{SPH}(\mathrm{F})$

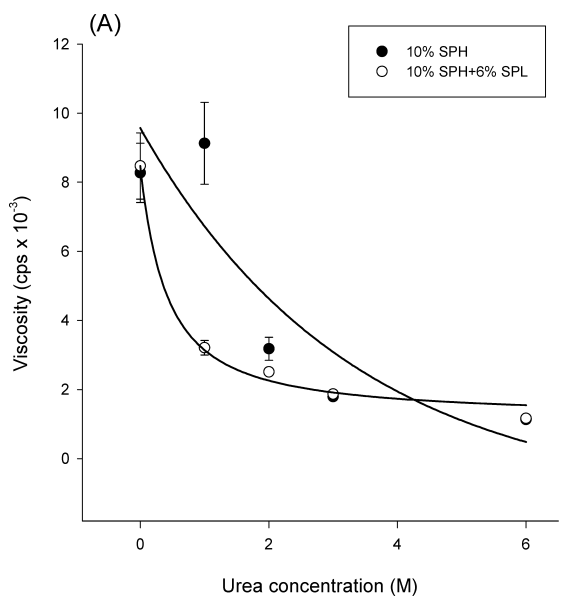

linking density as observed by the increased viscosity, and therefore decreased swelling and mean pore size, leading to slower drug release. ${ }^{17)}$ Although the Franz cell showed a limited loadong in receptor for buprenorphine, this method was still useful to differentiate the release capability of various formulations.

SEM analysis of the internal structure of the hydrogels showed a porous, fibrillar matrix which allowed the controlled transport of drug through the matrix. The amino acids in the SPH contain primary amines in their side chains, which are expected to be significantly protonated at $\mathrm{pH} 4$. Buprenorphine is a weak basic drug with a $\mathrm{p} K_{\mathrm{a}}$ of $8.24 .^{18)}$ The drug was almost completely positively ionized in the hydrogels. Hence buprenorphine cannot electrostatically interact with SPH in the hydrogels. Electrostatic binding can be excluded as the mechanism for the controlled release of buprenorphine. Other forces such as hydrodynamic drag, physical entanglement, and solute-hydrogel chemical interactions may be responsible for the observed reduced transport. Serine, aspartic acid, and glutamine, 3 main components in the $\mathrm{SPH}$, are all hydrophilic amino acids which may have an affinity for hydrophilic buprenorphine molecules, forming

Table 1. The Viscosity of Various Hydrogel Formulations

\begin{tabular}{lcr}
\hline \hline Polymer & $\begin{array}{c}\text { Concentration } \\
(\%, \mathrm{w} / \mathrm{v})\end{array}$ & $\begin{array}{c}\text { Viscosity } \\
\left(\mathrm{cps} \times 10^{-3}\right)\end{array}$ \\
\hline SPH $^{a)}$ & 8 & $1.73 \pm 0.19$ \\
SPH & 10 & $8.27 \pm 0.86$ \\
SPH & 12 & $12.69 \pm 1.43$ \\
SPH+SPL & $10: 2$ & $6.51 \pm 1.13$ \\
SPH+SPL & $10: 4$ & $8.33 \pm 1.01$ \\
SPH+SPL & $10: 6$ & $8.47 \pm 0.96$ \\
PF-127 $)$ & 20 & $35.06 \pm 0.55$ \\
PF-127+SPH & $20: 1$ & $46.86 \pm 1.23$ \\
PF-127+SPH & $20: 3$ & $53.76 \pm 0.80$ \\
PF-127+SPH & $20: 5$ & $63.90 \pm 0.37$ \\
Chitosan & 3 & $18.23 \pm 0.83$ \\
Chitosan+SPH & $3: 1$ & $20.12 \pm 0.31$ \\
Chitosan+SPH & $3: 3$ & $33.63 \pm 0.91$ \\
Chitosan+SPH & $3: 5$ & $57.58 \pm 1.69$ \\
Chitosan+SPH & $3: 10$ & $>66$ \\
\hline
\end{tabular}

a) SPH, silk protein high MW. b) SPL, silk protein low MW. c) PF-127, Pluronic F-127. Each value represents the mean \pm S.D. $(n=3)$.

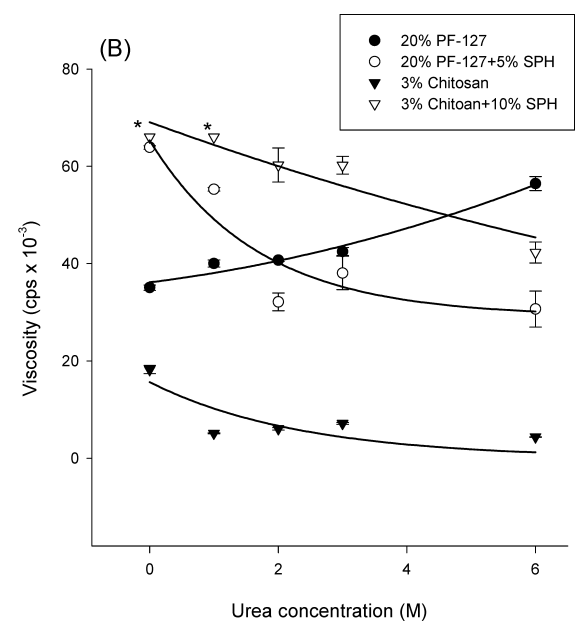

Fig. 4. Viscosity of SP Hydrogels (A) and PF-127/Chitosan Hydrogels with or without SPH (B) after Adding $0-6 \mathrm{M}$ Urea

$*$ Viscosity above the maximal detectable level ( $>66 \mathrm{kcps})$. Each value represents the mean \pm S.D. $(n=3)$. 

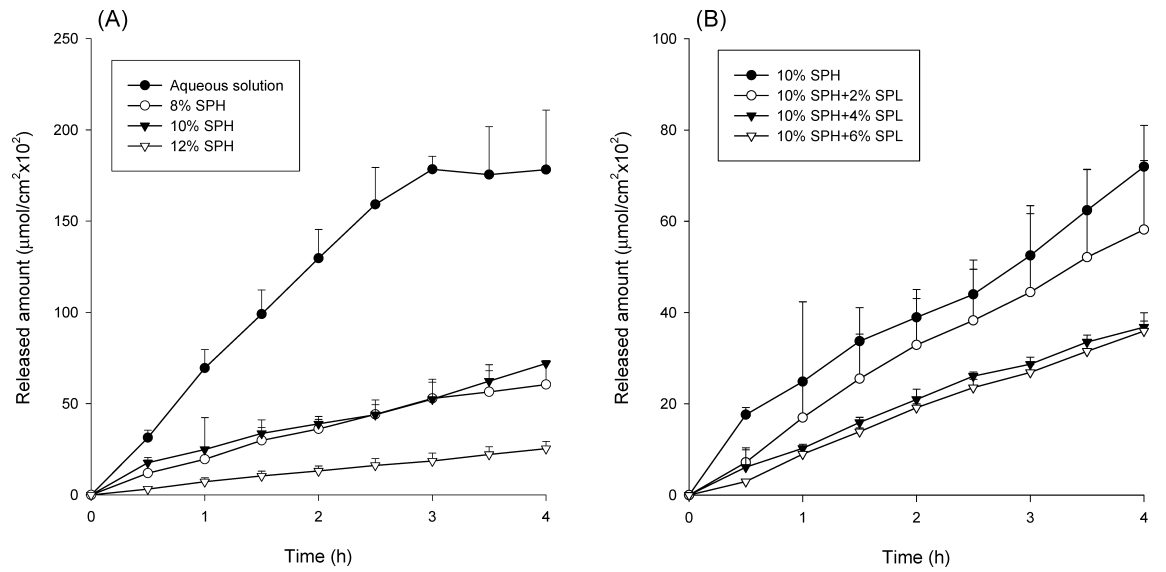

Fig. 5. Cumulative Amount-Time Profiles of Buprenorphine across Cellulose Membranes Released from Hydrogels Composed of SPH (A) and SPH/SPL (B) with Various Polymer Concentrations

Each value represents the mean \pm S.D. $(n=4)$.

Table 2. The Release Rate $\left(\mu \mathrm{mol} / \mathrm{cm}^{2} / \mathrm{h} \cdot 10^{2}\right)$ of Nalbuphine and Its Prodrug, FITC and FD from SP Hydrogels

\begin{tabular}{lcccc}
\hline \hline Formulation & Nalbuphine & Nalbuphine p. & FITC $^{a)}$ & FD $^{b)}$ \\
\hline Aqueous solution & $8.25 \pm 0.32$ & $5.72 \pm 0.40$ & $1208.23 \pm 65.77$ & $1806.42 \pm 112.53$ \\
SPH (10\%) & $1.71 \pm 0.12$ & $1.95 \pm 0.16$ & $32.08 \pm 3.00$ & $1104.82 \pm 60.16$ \\
SPH+SPL (10\%:6\%) & $1.58 \pm 0.09$ & $2.18 \pm 0.16$ & $20.23 \pm 3.02$ & $753.12 \pm 40.29$
\end{tabular}

a) FITC, fluorescein isothiocyanate. b) FD, FITC-labeled dextran. Each value represents the mean \pm S.D. $(n=4)$.

solute-hydrogel chemical interactions.

The incorporation of SPL further reduced the release rate of hydrogels composed of the SPH (Fig. 5B). The SPH hydrogels without SPL released a $c a .1 .4 \%$ dose to the receptor at the end of in vitro experiment $(4 \mathrm{~h})$. The release of buprenorphine generally decreased as the SPL concentration increased. No significant difference $(p>0.05)$ was observed between the release rate of SPH hydrogels with $4 \%$ SPL $\left(4.96 \pm 0.16 \mu \mathrm{mol} / \mathrm{cm}^{2} / \mathrm{h} \times 10^{2}\right)$ and $6 \%$ SPL $(4.93 \pm 0.33$ $\mu \mathrm{mol} / \mathrm{cm}^{2} / \mathrm{h} \times 10^{2}$ ). The hydrogen bonds which formed after blending the SPH and SPL may have further retarded the diffusion and elongated the release pathways of buprenorphine.

In order to explore the mechanisms of chemical interactions between the solute and hydrogel, other analgesics, nalbuphine and its prodrug, were introduced to examine their release profiles from SP hydrogels. Nalbuphine and nalbuphine propionate have approximately similar MWs (357.46 vs. 413.51, respectively). However, a large discrepancy was found in the octanol-water partition coefficient $(\log P, 0.17$ vs. 1.05, respectively). ${ }^{19)}$ As shown in Table 2, SPH hydrogels slowed the release of both nalbuphine and its prodrug compared to the aqueous solution. The aqueous solution could release nalbuphine and nalbuphine propionate for $c a$. $7.5 \%$ and $c a .5 \%$ of the total dose at the end of experiment. The reduced release was more significant for nalbuphine than for nalbuphine propionate. The incorporation of the SPL in SPH hydrogels did not further reduce the drug release. The more-significant reduction of nalbuphine release from the hydrogels suggests that the presence of attractive interactions between the solute and the polymer chains may be involved in the system. Nalbuphine and buprenorphine have a hydrophilic inherency in their structures. The dipole-dipole interactions between hydrophilic molecules and the SPH may have occurred which hindered release from the hydrogels. The longer ester side chain of nalbuphine propionate contributes to its higher lipophilicity. Hence the repulsive forces between the hydrophobic prodrug and the hydrophilic SPH may have occurred, leading to less retardation of prodrug release. Another explanation is based on the theory cited by Topp and colleagues ${ }^{20,21)}$ : lipophilic solutes such as nalbuphine propionate may diffuse through the hydrophobic as well as hydrophilic pathways of a hydrated polymeric environment. On the other hand, the hydrophilic nature of nalbuphine and buprenorphine suggests that their major diffusion pathway is only the hydrated portion of the hydrogels.

The size-dependent release of hydrogels was examined using FITC and FD as the solutes. SPH greatly slowed FITC release but not FD release from hydrogels (Table 2). The addition of SPL further reduced the release of both FITC and FD. This result is contrary to the obstruction theory of solute diffusion within hydrogels, ${ }^{10)}$ which assumes that the crosslinkage structure of hydrogels acts as a sieve allowing faster passage of smaller solutes than larger ones. The size-exclusion mechanism such as in Sephadex gels may explain the slower release of FITC than FD. As shown in Fig. 3A, both large and small pores exist in the structure of the SPH hydrogel system. The smaller pores admit small molecules into their interior but not large ones. The pathway inside smaller pores is more tortuous and slows down any solutes that enter. Solutes too large to enter the pathway simply by-pass the smaller pores, resulting in their rapid diffusion through the matrix. Hence the length of the diffusional paths is prominent in controlling drug release from SP hydrogels.

PF-127 and Chitosan Hydrogels Blended with SPs Blended systems of two independent polymers can be formed by cross-linking a second component within the 

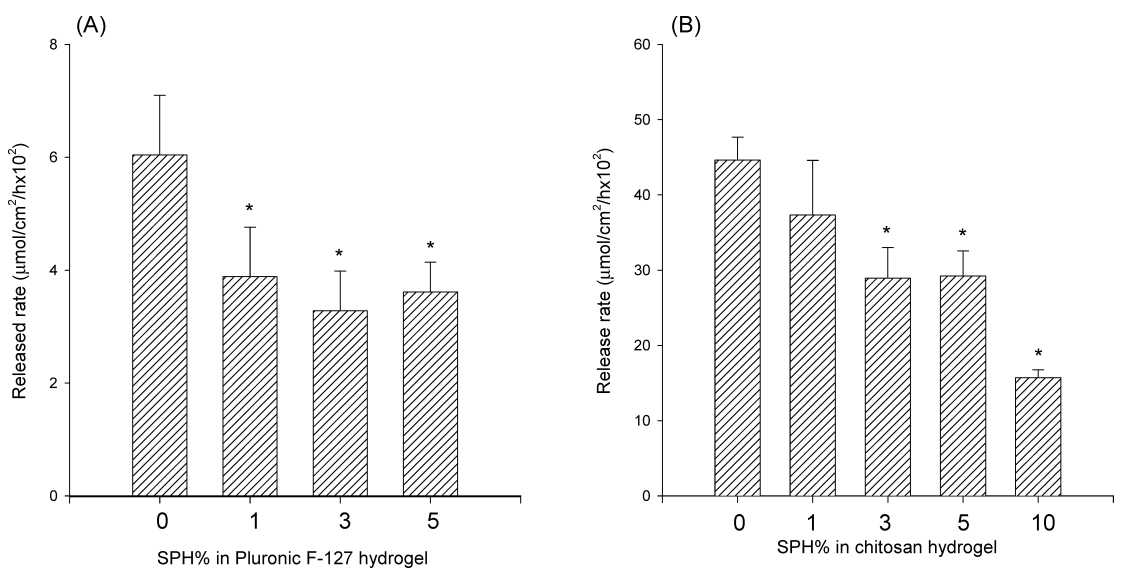

Fig. 6. Release Rate of Buprenorphine across Cellulose Membranes from Hydrogels Composed of PF-127/SPH (A) and Chitosan/SPH (B) with Various Polymer Concentrations

$* p<0.05$ compared to data of hydrogels without SPH. Each value represents the mean \pm S.D. $(n=4)$.

cross-linked hydrophilic network. Advantages of the blended system include the formation of a mechanically stronger hydrogel and an increase in the biocompatibility. ${ }^{14)}$ The other two polymers, PF-127 and chitosan, were therefore combined with SPH to produce new hydrogel systems. PF-127 is a surface-active block copolymer of polyoxyethylene-polyoxypropylene which has a MW of $12.6 \mathrm{kDa}$. PF-127 is used for drug delivery due to its good biocompatibility and rapidly reversible sol-gel transition behavior with respect to temperature and concentration. ${ }^{14)}$ Chitosan is an alkaline deacetylated product from the living crustacean exoskeleton. This natural, cationic polysaccharide possesses great properties such as non-toxicity, high biocompatibility, and nonantigenicity. ${ }^{22)}$

Figure 2B shows that the FTIR spectra of PF-127 and chitosan hydrogels ranged from 1200 to $1700 \mathrm{~cm}^{-1}$. A 20\% PF127 was used because gel formation was not observed below this concentration. The bands at 1350 and $1456 \mathrm{~cm}^{-1}$ were respectively assigned to $-\mathrm{CH}_{3}$ and $-\mathrm{CH}_{2}-$ stretching of he PF-127 chain. The chitosan hydrogel showed absorption bands at 1317 and $1575 \mathrm{~cm}^{-1}$, which were respectively attributed to the amide group of chitin and the amino group of chitosan. The band at $1412 \mathrm{~cm}^{-1}$ was assigned to the $\mathrm{C}-\mathrm{N}$ group of the chitosan chain. The blend of the SPH with PF-127 or chitosan retained all of the absorption bands of the individual polymer in hydrogels. The absorption at 1635 and $1650 \mathrm{~cm}^{-1}$ (amide I) for PF-127/SPH and chitosan/SPH hydrogels respectively indicate the presence of both the $\beta$-structure and random coil in the blended gels (Fig. 2B).

When PF-127 was dissolved in an aqueous solution, the copolymer molecules aggregated into micelles with deformed shapes (Fig. 3C). ${ }^{23}$ ) As shown in Fig. 3D, a tightly orientated scaffold structure was formed within the PF-127 micellar pathways by SPH entanglement. Chitosan formed cross-linked network gels with interconnecting pores in the freeze-dried form (Fig. 2E). The network structure of chitosan was more condensed than that of SPH (Fig. 3A). This result may be attributed to the higher viscosity of chitosan than the SPH formulations (Table 1). After incorporating a $10 \% \mathrm{SPH}$ into the chitosan hydrogel, the matrix showed sheets of smooth interconnecting material with smaller channels.
As shown in Table 1, the high viscosity of PF-127 hydrogels reflects the viscous isotropic liquid crystals comprising the micelles. ${ }^{24)} \mathrm{A}$ linear increase in viscosity following an increase of the SPH ( $0 \%$ to $5 \%)$ in PF-127 hydrogels was detected $(r=0.98)$. A $10 \%$ SPH was also blended with the PF127 hydrogel. However, data of this formulation were excluded because of the incomplete solubilization of the $10 \%$ $\mathrm{SPH}$ in the system. An increased percentage of SPH in the chitosan hydrogel resulted in a steeper increase in viscosity $(r=0.95)$ compared to PF-127. This may indicate that a strong chemical interaction or physical entanglement was generated between chitosan and the SPH. The role of hydrogen binding in the blended systems was also examined by adding urea to the gels. As shown in Fig. 4B, the addition of urea at various concentrations gradually increased the viscosity of PF-127 hydrogels. This suggests the limited role of hydrogen binding in the PF-127 system. The gradual increase in viscosity may have been caused by the urea itself. Contrary to this result, the viscosity gradually decreased after the addition of urea to PF-127/SPH hydrogels. This suggests that the cross-linked texture formed after incorporation of the SPH in PF-127 hydrogels resulted from physical entanglement and extensive hydrogen binding. Urea at various concentrations $(1-6 \mathrm{M})$ reduced the chitosan hydrogel viscosity to a similar level (Fig. 4B). This indicates that $1 \mathrm{~m}$ urea was sufficient to break all hydrogen bonds in the chitosan hydrogels. Covalent bonds are the main interactions forming the chitosan networks. Incorporation of the SPH in the chitosan systems increased the hydrogen binding interactions since the viscosity gradually decreased with the addition of urea. A previous study suggested that when another polymer is included, additional secondary interactions between this polymer and chitosan arise such as intermolecular hydrogen bonds. ${ }^{25}$

PF-127 hydrogels retarded buprenorphine release to the level of inhibition of SPH hydrogels $(10 \%)(p>0.05$, Fig. 6A). Drug diffusion from PF-127 gels occurs through extramicellar aqueous channels, and the microviscosity of the water channels controls the release. ${ }^{4)}$ When the cross-linking structure was formed after incorporating the $\mathrm{SPH}$, the buprenorphine release was further reduced from PF-127 vehicles $(p<0.05)$. The blending of another polymer into the 
PF-127 gels decreased the amount of free water in the aqueous channels and affected the molecular orientation of the gel matrix. There was no significant difference $(p>0.05)$ among the amounts of buprenorphine released with different SPH concentrations $(1-5 \%$, Fig. 6A). A higher viscosity was detected with higher SPH concentrations in PF-127 systems, inferring a more-condensed matrix in the vehicles. This may indicate that the further entanglement of the matrix did not affect the length or size of the diffusional paths for buprenorphine.

Although chitosan hydrogels possessed a more-condensed network texture than the SPH, they showed limited decreases in buprenorphine release compared to the aqueous solution (Fig. 6B). A possible reason is that the chitosan backbone has a high positive charge density. ${ }^{22)}$ The repulsion forces between the cationic buprenorphine and chitosan polymer may promote the escape of the drug by diffusion from the system. Incorporation of the SPH at various concentrations into chitosan hydrogels resulted in a retarded release of buprenorphine, although no significant difference $(p>0.05)$ was detected between the release rate of vehicles with $0 \%$ and $1 \%$ SPH (Fig. 5B). A linear relationship $(r=0.96)$ between the release rate and percent SPH was observed. This phenomenon may have been related to greater polymer entanglement and lower effective molecular diffusion area as the SPH concentration increased.

\section{Conclusions}

SPH (MW ca. $76 \mathrm{kDa}$ ) and SPL (MW ca. $18 \mathrm{kDa})$ are SP polymers composed of abundant hydrophilic amino acids. Both $\beta$-sheet and random coils existed in the conformation of SP hydrogels. SPH hydrogels formed an interconnecting texture with pores. Further incorporation of SPL into SPH hydrogels changed the cross-linking structure to a sheet shape. Hydrogen binding is important for the linkage of hydrogels. The in vitro release experiments indicated that SPH or SPH/SPL hydrogels can serve as a rate-controlling barrier and may be useful as a vehicle for sustained-release preparations of buprenorphine. The drug release from SPH hydrogels was comparable to that from PF-127 hydrogels and was slower than that from chitosan hydrogels. Since SPH showed lower viscosity than the other polymers, it is feasible for parenteral administration because of the ease to send out from the injections. Changes in the SPH and SPL concentrations in hydrogels can further control drug release rates. Incorporation of the SPH in PF-127 and chitosan hydrogels further reduced the release of buprenorphine from the systems.

\section{References}

1) Kim U. J., Park J., Li C., Jin H. J., Valluzzi R., Kaplan D. L., Biomacromolecules, 5, 786-792 (2004).

2) Altman G. H., Diaz F., Jakuba C., Calabro T., Horan R. L., Chen J., Lu H., Richmond J., Kaplan D. L., Biomaterials, 24, 401- 416 (2003).

3) Cascone M. G., Sim B., Bownes S., Biomaterials, 16, 569-574 (1995).

4) Paavola A., Yliruusi J., Rosenberg P., J. Control. Release, 52, 169178 (1998)

5) Dinerman A. A., Cappello J., Chandehari H., Hoag S. W., J. Control. Release, 82, 277-287 (2002).

6) Imoto H., Zhou Z., Stinchcomb A. L., Flynn G. L., Biol. Pharm. Bull., 19, 263-267 (1996).

7) Fang J. Y., Sung K. C., Wang J. J., Chu C. C., Chen K. T., J. Pharm. Pharmacol., 54, 1329-1337 (2002)

8) Wilding I. R., Davis S. S., Rimoy G. H., Rubin P., Kurihara-Bergstrom T., Tipnis V., Berner B., Nightingale J., Int. J. Pharm., 132, 81-87 (1996).

9) Sung K. C., Fang J. Y., Hu O. Y. P., J. Control. Release, 67, 1-8 (2000).

10) Amsden B., Macromolecules, 31, 8382-8395 (1998).

11) Kim S. W., Bae T. H., Okano T., Pharm. Res., 9, 283-290 (1992).

12) Narasimhan B., Peppas N. A., J. Pharm. Sci., 86, 297-304 (1997)

13) Kweon H. Y., Ha H. C., Um I. C., Park Y. H., J. Appl. Polym. Sci., 80, 928-934 (2001).

14) Yoo M. K., Kweon H. Y., Lee K. G., Lee H. C., Cho C. S., Int. J. Biol. Macromol., 34, 263-270 (2004).

15) Cappello J., Crissman J. W., Crissman M., Ferrari F. A., Textor G., Wallis O., Whitledge J. R., Zhou X., Burman D., Aukerman L., Stedronsky E. R., J. Control. Release, 53, 105-117 (1998).

16) Kang G. D., Nahm J. H., Park J. S., Moon J. Y., Cho C. S., Yeo J. H., Macromol. Rapid Commun., 21, 788-791 (2000).

17) Mageed Z., Cappello J., Ghandehari H., Pharm. Res., 19, 954-959 (2002).

18) Roy S. D., Roos E., Sharma K., J. Pharm. Sci., 83, 126-130 (1994).

19) Sung K. C., Fang J. Y., Wang J. J., Hu O. Y. P., Eur. J. Pharm. Sci., 18, $63-70$ (2003).

20) Joshi H. N., Topp E. M., Int. J. Pharm., 80, 213-221 (1992).

21) Sung K. C., Topp E. M., J. Control. Release, 37, 95-104 (1995).

22) Singla A. K., Chawla M., J. Pharm. Pharmacol., 53, 1047-1067 (2001).

23) Moore T., Croy S., Mallapragada S., Pandit N., J. Control. Release, 67, 191-202 (2000).

24) Wang Y. Y., Hong C. T., Chiu W. T., Fang J. Y., Int. J. Pharm., 224, 89-104 (2001).

25) Berger J., Reist M., Mayer J. M., Felt O., Peppas N. A., Gurny R., Eur J. Pharm. Biopharm., 57, 19-34 (2004). 